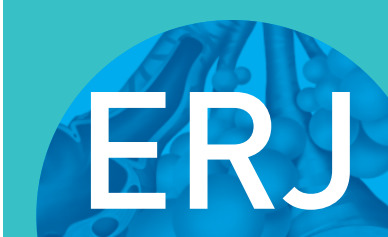

open research

\title{
Critical care outcomes in patients with pre-existing pulmonary hypertension: insights from the ASPIRE registry
}

\author{
Kris Bauchmuller (10 ${ }^{1,4}$, Robin Condliffe (10 ${ }^{2,3,4}$, Jennifer Southern ${ }^{1,4}$, \\ Catherine Billings ${ }^{2}$, Athanasios Charalampopoulos ${ }^{2}$, Charlie A. Elliot ${ }^{2}$,

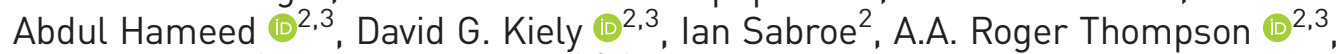 \\ Ajay Raithatha ${ }^{1}$ and Gary H. Mills ${ }^{1,3,4}$
}

Affiliations: ${ }^{1}$ Dept of Critical Care, Sheffield Teaching Hospitals NHS Foundation Trust, Sheffield, UK ${ }^{2}$ Sheffield Pulmonary Vascular Disease Unit, Sheffield Teaching Hospitals NHS Foundation Trust, Sheffield, UK. ${ }^{3}$ Dept of Infection, Immunity and Cardiovascular Disease, University of Sheffield, Sheffield, UK. ${ }^{4}$ These authors contributed equally.

Correspondence: Kris Bauchmuller, Dept of Critical Care and Anaesthesia, Sheffield Teaching Hospitals NHS Foundation Trust, Glossop Road, Sheffield, S10 2JF, UK. E-mail: Kris.bauchmulleranhs.net

ABSTRACT Pulmonary hypertension (PH) is a life-shortening condition characterised by episodes of decompensation precipitated by factors such as disease progression, arrhythmias and sepsis. Surgery and pregnancy also place additional strain on the right ventricle. Data on critical care management in patients with pre-existing $\mathrm{PH}$ are scarce.

We conducted a retrospective observational study of a large cohort of patients admitted to the critical care unit of a national referral centre between 2000-2017 to establish acute mortality, evaluate predictors of in-hospital mortality and establish longer term outcomes in survivors to hospital discharge.

242 critical care admissions involving 206 patients were identified. Hospital survival was 59.3\%, 94\% and $92 \%$ for patients admitted for medical, surgical or obstetric reasons, respectively. Medical patients had more severe physiological and laboratory perturbations than patients admitted following surgical or obstetric interventions. Higher APACHE II (Acute Physiology and Chronic Health Evaluation) score, age and lactate, and lower oxygen saturation measure by pulse oximetry/inspiratory oxygen fraction $\left(S_{\mathrm{pO}_{2}} / F_{\mathrm{iO}_{2}}\right)$ ratio, platelet count and sodium level were identified as independent predictors of hospital mortality. An exploratory risk score, OPALS (oxygen $\left(S_{\mathrm{pO}_{2}} / F_{\mathrm{iO}_{2}}\right) \leqslant 185$; platelets $\leqslant 196 \times 10^{9} \cdot \mathrm{L}^{-1}$; age $\geqslant 37.5$ years; lactate $\geqslant 2.45 \mathrm{mmol} \cdot \mathrm{L}^{-1}$; sodium $\left.\leqslant 130.5 \mathrm{mmol} \cdot \mathrm{L}^{-1}\right)$, identified medical patients at increasing risk of hospital mortality. One (11\%) out of nine patients who were invasively ventilated for medical decompensation and $50 \%$ of patients receiving renal replacement therapy left hospital alive. There was no significant difference in exercise capacity or functional class between follow-up and pre-admission in patients who survived to discharge.

These data have clinical utility in guiding critical care management of patients with known $\mathrm{PH}$. The exploratory OPALS score requires validation.

@ERSpublications

Critical care survival is worse in PH patients admitted for medical rather than surgical/obstetric indications. Nevertheless, many show longer term survival and functional recovery. Markers of severity of acute illness at admission are prognostic. https://bit.ly/2YX9Fw9

Cite this article as: Bauchmuller K, Condliffe R, Southern J, et al. Critical care outcomes in patients with pre-existing pulmonary hypertension: insights from the ASPIRE registry. ERJ Open Res 2021; 7: 00046-2021 [https://doi.org/10.1183/23120541.00046-2021].

Received: 20 Jan 2021 | Accepted: 31 Jan 2021

Copyright $\odot$ The authors 2021. This version is distributed under the terms of the Creative Commons Attribution NonCommercial Licence 4.0. For commercial reproduction rights and permissions contact permissions@ersnet.org 


\section{Introduction}

Pulmonary hypertension $(\mathrm{PH})$ describes a group of conditions characterised by the presence of a mean pulmonary arterial pressure $\geqslant 25 \mathrm{mmHg}$ [1]. Increase in right ventricular (RV) afterload results in right ventricular impairment with subsequent reduced exercise capacity, RV failure and premature death $[2,3]$. Pulmonary vasodilator medical therapy and pulmonary endarterectomy surgery have been demonstrated to improve long-term outcomes in patients with group 1 (pulmonary arterial hypertension; PAH) and group 4 disease (chronic thromboembolic PH; CTEPH), respectively $[4,5]$.

Despite these advances in treatment, $\mathrm{PH}$ is still a life-shortening condition with a propensity for episodes of decompensation precipitated by factors such as disease progression, arrhythmias and sepsis [6, 7]. Surgery and pregnancy also place additional strain on the RV and are associated with increased mortality and morbidity [8-10]. As such, patients with $\mathrm{PH}$ may require treatment on the critical care unit during the course of their disease [6]. There are relatively few published papers regarding critical care outcomes for $\mathrm{PH}$ patients. These are often limited by sample size [11-14] or lack of data granularity [15].

We therefore conducted a retrospective observational cohort study of patients with known pre-existing $\mathrm{PH}$ who had been admitted to the critical care unit (encompassing patients requiring general high dependency or general intensive care beds) of a UK PH referral centre over a 17-year period, to establish acute mortality, evaluate outcome predictors and define longer term outcomes.

\section{Methods}

\section{Study design}

Consecutive patients (aged $\geqslant 16$ years) in the ASPIRE registry [16] who had been managed on our critical care units between April 2000 and December 2017 were identified by cross-referencing with two critical care databases: the critical care clinical information system (MetaVision ICU; iMDsoft, Tel Aviv, Israel) and the local Intensive Care National Audit and Research Centre case mix programme database. $\mathrm{PH}$ was categorised according to the 5th World Symposium on Pulmonary Hypertension classification [17]. The study was approved by both the UK Health Research Authority (HRA, IRAS no 246341) and the local institutional review board (STH 20394) after independent scientific review.

\section{Data}

Critical care data collected included: patient demographics; admission circumstances (medical/surgical/ obstetric; planned versus unplanned); Acute Physiology and Chronic Health Evaluation II score (APACHE II); routine physiological and laboratory parameters; treatment modalities (vasopressors, inotropes, noninvasive ventilatory support, invasive positive pressure ventilation, renal replacement therapy (RRT)); critical care and hospital length of stay; and critical care and hospital mortality. APACHE II score and physiological and laboratory parameters were taken at critical care admission. Longer term survival status was ascertained at the censoring date of 31 December 2018 using data from the NHS Personal Demographics Service to ensure at least 1-year follow-up for survivors. Where survival allowed it, follow-up included 5-year data and beyond. In addition, we retrieved the following data from the ASPIRE registry: PH classification, right heart catheter data (most recent prior to admission), World Health Organization (WHO) functional class (FC) and incremental shuttle walk distance (ISWD) at baseline (2 weeks to 18 months prior to admission) and at follow-up (nearest to 12 months post-discharge for WHO FC, and nearest to 6 and 12 months post-discharge for ISWD).

\section{Statistical analysis}

Data were analysed using SPSS Statistics version 22. Quantitative data are described as mean \pm SD or median (range or interquartile range) as appropriate. Normality of data was assessed using histograms and the Kolmogorov-Smirnov test. Categorical data were analysed using the Chi-squared and Fisher's exact test. The paired samples t-test and related-samples Wilcoxon signed rank test were used to assess differences in ISWD and WHO FC over time. Differences in length of stay between groups were examined with the Kruskal-Wallis method. The association between admission characteristics and mortality was assessed using univariate and multiple-variable logistic regression analysis. The latter was conducted with a hierarchical approach using a pre-determined limited number of variables based on their biological and clinical plausibility in light of the existing evidence base, having removed those with high collinearity or significant missing data. Survival analysis was performed using the Kaplan-Meier method, log-rank testing and Cox regression. Only first presentations to the intensive care unit (ICU) were taken into account for survival analysis. Results are expressed as odds ratios or hazard ratios with $95 \%$ confidence intervals as appropriate. Receiver operating characteristic analysis was used to identify optimal thresholds for hospital survival. An exploratory risk score for medical patients, based on the number of adverse parameters using these thresholds, was subsequently calculated. A p-value $<0.05$ was regarded as statistically significant throughout. 


\section{Results}

\section{Baseline characteristics}

Overall, 242 consecutive critical care admissions in 206 individual patients were included in the study. The median (min-max) age of the study population was 52.5 (17-87) years and 68\% of patients were female. The majority of patients had PAH (67\%) or CTEPH (16\%). Baseline characteristics are summarised in table 1. 167 (69\%) admissions were for medical reasons, followed by surgical and obstetric indications in $50(21 \%)$ and $25(10 \%)$ admissions, respectively. The commonest reasons for medical admissions were right heart failure $(38 \%)$ and respiratory failure and/or respiratory infection $(26 \%)$. The majority of surgical patients $(84 \%)$ were elective admissions. Medical patients had a greater degree of physiological and biochemical derangement on critical care admission compared with the surgical and obstetric cohorts, exemplified by higher APACHE II scores, poorer oxygenation, worse renal and liver biochemistry and higher C-reactive protein. Detailed patient characteristics on critical care admission are provided in table 2.

\section{Acute mortality and length of stay}

Overall, critical care and hospital survival rates were $78.5 \%$ and $69.8 \%$, respectively. Medical patients had lower hospital survival (59\%) compared with their surgical (94\%) or obstetric counterparts (92\%; $<<0.001$ for medical versus surgical groups and $\mathrm{p}=0.006$ for medical versus obstetric groups) (table 3 ). Crude critical care, and hospital, 90-day and 1-year survival rates according to patient group, $\mathrm{PH}$ subtype and critical care-specific therapies are provided in table 3. Unplanned admissions had lower hospital survival than planned admissions (61.0\% versus $93.8 \%, \mathrm{p}<0.001)$. The median (min-max) length of stay in critical care for medical, surgical and obstetric patients was 3.6 (0.02-36.9), $1.5(0.7-10.8)$ and $3.2(0.7-9.0)$ days, respectively. The median $(\min -\max )$ in-hospital length of stay (from critical care admission) for these patient groups was $11.3(0.02-108.0), 7.4(0.9-68.2)$ and 9.0 (1.3-16.0) days. Medical patients had significantly longer stays on critical care $(\mathrm{p}=0.004)$ and in hospital $(\mathrm{p}=0.041)$ when compared with their surgical counterparts.

\begin{tabular}{|c|c|}
\hline Subjects $\mathrm{n}$ & 242 \\
\hline Age years & $52.5(17-87)$ \\
\hline Female & 68.2 \\
\hline \multicolumn{2}{|l|}{ PH diagnostic group } \\
\hline 1 Pulmonary arterial hypertension & $162(66.9)$ \\
\hline Idiopathic/heritable/drugs and toxins & 96 (59.3) \\
\hline Connective tissue disease associated & 40 (24.7) \\
\hline Congenital heart disease associated & $18(11.1)$ \\
\hline Others & $8(4.9)$ \\
\hline $2 \mathrm{PH}$ due to left heart disease & $13(5.3)$ \\
\hline $3 \mathrm{PH}$ due to lung disease/chronic hypoxia & $14(5.8)$ \\
\hline 4 Chronic thromboembolic $\mathrm{PH}$ & $39(16.1)$ \\
\hline 5 Unclear/multifactorial & $14(5.8)$ \\
\hline \multicolumn{2}{|l|}{ WHO functional class $\#$} \\
\hline । & 1.4 \\
\hline II & 14.9 \\
\hline III & 70.2 \\
\hline IV & 13.5 \\
\hline ISWD m" & $190(0-1020)$ \\
\hline \multicolumn{2}{|l|}{ Pulmonary haemodynamics" } \\
\hline mRAP $\mathrm{mmHg}$ & $10.0(1-34)$ \\
\hline $\mathrm{mPAP} \mathrm{mmHg}$ & $47(14-74)$ \\
\hline PAWP $\mathrm{mmHg}$ & $10(0-33)$ \\
\hline $\mathrm{CO} L \cdot \mathrm{min}^{-1}$ & $4.7(1.87-14.2)$ \\
\hline $\mathrm{Cl} \mathrm{L} \cdot \mathrm{min}^{-1} \cdot \mathrm{m}^{-2}$ & $2.6(1.2-6.0)$ \\
\hline PVR dyn $\cdot \mathrm{s}^{-1} \cdot \mathrm{cm}^{-5}$ & $561(84-2400)$ \\
\hline $\mathrm{S}_{\mathrm{vO}_{2}} \%$ & $65(35-86)$ \\
\hline
\end{tabular}

Data are presented as median (min-max), \% or $\mathrm{n}(\%)$, unless otherwise stated. $\mathrm{PH}$ : pulmonary hypertension; WHO: World Health Organization; ISWD: incremental shuttle walk distance; mRAP: mean right atrial pressure; mPAP: mean pulmonary artery pressure; PAWP: pulmonary arterial wedge pressure; $\mathrm{CO}$ : cardiac output; $\mathrm{Cl}$ : cardiac index; PVR pulmonary vascular resistance; $\mathrm{S}_{\mathrm{vo}_{2}}$ : mixed venous oxygen saturations. " : 2 weeks to 18 months prior to admission; " : nearest prior to admission. 


\section{TABLE 2 Patient characteristics on critical care admission}

\section{Subjects}

\begin{tabular}{|c|c|c|c|}
\hline \multicolumn{4}{|l|}{ Patient group } \\
\hline Medical & $167(69.0)$ & & \\
\hline Surgical & $50(20.7)$ & & \\
\hline Obstetric & 25 (10.3) & & \\
\hline \multicolumn{4}{|l|}{ Main reason for admission } \\
\hline \multicolumn{4}{|l|}{ Medical patients } \\
\hline Isolated heart failure & 63 (37.7) & & \\
\hline Respiratory failure/chest sepsis & $44(26.4)$ & & \\
\hline Sepsis lother) & $13(7.8)$ & & \\
\hline Renal failure & $12(7.2)$ & & \\
\hline Arrhythmia & $14(8.4)$ & & \\
\hline PE & $6(3.6)$ & & \\
\hline Other & $15(9)$ & & \\
\hline \multicolumn{4}{|l|}{ Surgical patients } \\
\hline Peri-operative (planned): & $42(84)$ & & \\
\hline Orthopaedic & $18(36)$ & & \\
\hline Hernia repair & $4(8)$ & & \\
\hline Other general surgery & 7 (14) & & \\
\hline Urological & $3(6)$ & & \\
\hline Miscellaneous $\#$ & $10(20)$ & & \\
\hline Peri-operative (emergency): & $8(16)$ & & \\
\hline Urological & $3(6)$ & & \\
\hline Neurosurgical & $2(4)$ & & \\
\hline General & $1(2)$ & & \\
\hline Gynaecological & $1(2)$ & & \\
\hline Orthopaedic & $1(2)$ & & \\
\hline \multicolumn{4}{|l|}{ Obstetric patients } \\
\hline Peri-partum & $19(76)$ & & \\
\hline Other pregnancy related causes & $6(24)$ & & \\
\hline Physiological parameters & Medical" & Surgical/obstetric $^{+}$ & p-value \\
\hline APACHE II & $15.0(2-31)$ & $10(2-21)$ & $<0.001$ \\
\hline Heart rate & $99(48-163)$ & $80(49-126)$ & $<0.001$ \\
\hline $\mathrm{mSAP} \mathrm{mmHg}$ & $79(41-140)$ & $85(56-117)$ & 0.001 \\
\hline Respiratory rate & $24(12-52)$ & $17(9-39)$ & $<0.001$ \\
\hline $\mathrm{S}_{\mathrm{pO}_{2}} / \mathrm{F}_{\mathrm{iO}_{2}}$ ratio & $168(68-467)$ & $278(108-467)$ & $<0.001$ \\
\hline$P_{\mathrm{aO}_{2}} \mathrm{kPa}$ & $10.4(4.3-28.9)$ & $13.7(6.9-25.3)$ & $<0.001$ \\
\hline$P_{\mathrm{aO}_{2}} / \mathrm{F}_{\mathrm{iO}_{2}}$ ratio & $22(5-72)$ & $49(9-89)$ & $<0.001$ \\
\hline CVP $\mathrm{mmHg}$ & $18(2-35)$ & $8(3-22)$ & $<0.001$ \\
\hline Glasgow Coma Scale & 15 (3-15) & $15(13-15)$ & 0.010 \\
\hline $\mathrm{pH}$ & $7.43(6.98-7.54)$ & $7.41(7.31-7.51)$ & 0.253 \\
\hline Base excess $\mathrm{mmol} \cdot \mathrm{L}^{-1}$ & $-2.6(-20.4-26.9)$ & $-1.75(-8.5-7.2)$ & 0.219 \\
\hline Lactate $\mathrm{mmol} \cdot \mathrm{L}^{-1}$ & $1.2(0.4-14.1)$ & $1.0(0.5-2.4)$ & 0.006 \\
\hline Sodium $\mathrm{mmol} \cdot \mathrm{L}^{-1}$ & 135 (113-147) & $138(127-146)$ & $<0.001$ \\
\hline Urea $\mathrm{mmol} \cdot \mathrm{L}^{-1}$ & $10.2(2.5-59.4)$ & $3.9(1.2-16.1)$ & $<0.001$ \\
\hline Creatinine $\mu \mathrm{mol} \cdot \mathrm{L}^{-1}$ & $117(3-891)$ & $61.5(26-181)$ & $<0.001$ \\
\hline Bilirubin $\mu \mathrm{mol} \cdot \mathrm{L}^{-1}$ & $17(1-131)$ & $11(2-55)$ & $<0.001$ \\
\hline AST IU.L $L^{-1}$ & $27(6-4733)$ & $18(11-150)$ & $<0.001$ \\
\hline CRP $\mathrm{mg} \cdot \mathrm{L}^{-1}$ & $38(1-471)$ & $11.6(0.3-340)$ & $<0.001$ \\
\hline Haemoglobin $\mathrm{g} \cdot \mathrm{L}^{-1}$ & 119 (53-204) & $113(86-166)$ & 0.027 \\
\hline Platelets $\times 10^{9} \cdot \mathrm{L}^{-1}$ & $194(32-859)$ & $202(73-448)$ & 0.297 \\
\hline
\end{tabular}

Data are presented as $\mathrm{n}(\%)$ or median (min-max), unless otherwise stated. Comparisons made using Mann-Whitney U-test, medical versus surgical/obstetric groups. PE: pulmonary embolism; APACHE II: Acute Physiology and Chronic Health Evaluation II; mSAP: mean systemic arterial pressure; $S_{\mathrm{pO}_{2}}$ : oxygen saturation measured by pulse oximetry; $F_{\mathrm{iO}_{2}}$ : inspiratory oxygen fraction; $P_{\mathrm{aO}}$ : arterial oxygen tension; CVP: central venous pressure; AST: aspartate aminotransferase; CRP: C-reactive protein. \# : neurosurgery, spinal surgery, vascular, breast and gynaecological; ${ }^{\uparrow}: n=167 ;^{+}: n=75$. 
TABLE 3 Critical care and hospital survival according to patient group and intensive care unit (ICU) therapy

N ICU survival Hospital survival 90-day survival 1-year survival $\mathrm{N}^{\#}$ 5-year survival ${ }^{\#}$

\begin{tabular}{|c|c|c|c|c|c|c|c|}
\hline Overall & 242 & $190(78.5)$ & 169 (69.8) & $158(65.3)$ & $132(54.5)$ & 146 & 45 (30.8) \\
\hline \multicolumn{8}{|l|}{ Patient group } \\
\hline Medical & 167 & $120(71.9)$ & 99 (59.3) & $91(54.5)$ & $68(40.7)$ & 114 & $26(22.8)$ \\
\hline Surgical & 50 & $47(94.0)$ & 47 (94.0) & $45(90)$ & $42(84)$ & 19 & $10(52.6)$ \\
\hline \multicolumn{8}{|l|}{ PH group } \\
\hline $1 \mathrm{PAH}$ & 162 & 127 (78.4) & 115 (71.0) & $107(66.0)$ & $90(55.6)$ & 95 & 32 (33.7) \\
\hline $2 \mathrm{PH}$ due to left heart disease & 13 & $11(84.6)$ & 10 (76.9) & 10 (76.9) & $8(61.5)$ & 7 & $2(28.6)$ \\
\hline 5 Unclear/multifactorial & 14 & $9(64.3)$ & $7(50)$ & $7(50)$ & $4(28.6)$ & 8 & $1(12.5)$ \\
\hline \multicolumn{8}{|l|}{ ICU therapy (medical patients only) } \\
\hline CPAP as highest level of ventilatory support & 53 & $34(64.1)$ & $27(50.9)$ & $25(47.1)$ & 20 (37.7) & 33 & $2(6.1)$ \\
\hline NPPV as highest level of ventilatory support & 15 & $9(60)$ & $6(40)$ & 5 (33.3) & $3(20)$ & 10 & $0(0)$ \\
\hline IPPV as highest level of ventilatory support & 9 & 3 (33.3) & $1(11.1)$ & $1(11.1)$ & $1(11.1)$ & 9 & $0(0)$ \\
\hline CRRT & 22 & $17(77.3)$ & $11(50.0)$ & $9(40.9)$ & $7(31.8)$ & 20 & $5(25)$ \\
\hline
\end{tabular}

Data are presented as $\mathrm{n}(\%)$, unless otherwise stated. $\mathrm{PH}$ : pulmonary hypertension; PAH: pulmonary arterial hypertension; CPAP: continuous positive airway pressure; NPPV: non-invasive positive pressure ventilation: IPPV: invasive positive pressure ventilation; CRRT: continuous renal replacement therapy. " : patients admitted prior to 31 December 2013 for whom 5 years of survival data is available.

Although a number of patients were on an active lung or heart/lung transplant list at the time of critical admission, no patients were transferred directly for transplantation (with or without extracorporeal membrane oxygenation). Hospital mortality was worse in the 48 medical admissions during 2000-2010 compared with the 119 patients admitted subsequently $(65 \%$ versus $31 \%, \mathrm{p}<0.001)$. Although there was no significant difference in age or most recent ISWD and FC between these two groups, median APACHE II score was significantly higher in the earlier group (19 (range 16-22) versus 14 (10-19) in the latter group ( $\mathrm{p}=0.005)$ ).

\section{Predictors of hospital mortality in the medical group}

Univariate analysis for medical patients demonstrated a significant association with hospital mortality for age, primary admission reason, APACHE II score and several physiological and biochemical markers on admission (table 4). However, measures of pre-admission PH severity and functional state were not significantly associated with acute mortality. In multivariate analysis of the medical patient cohort, higher APACHE II score and lower serum sodium level were independent predictors of hospital mortality (Model 1) (table 5). Since APACHE II score incorporates several of the selected variables and may therefore mask their individual contribution, analysis was repeated (Model 2) (table 5) without APACHE II. Higher age and serum lactate, and lower pulse oximetry/inspiratory oxygen fraction $\left(S_{\mathrm{pO}_{2}} /\right.$ $\left.F_{\mathrm{iO}_{2}}\right)$ ratio and platelet count gained significance while low sodium retained its significant association with mortality. When multivariate analysis was repeated for the entire study cohort (including surgical and obstetric patients), the results mirrored those of the medical group alone (data for entire study group not shown).

In-hospital mortality for medical patients, according to quintiles of the independent prognostic markers is shown in figure 1. Area under the curve (AUC) and optimal threshold for predicting hospital mortality in the medical patient group for each parameter were: APACHE II: 0.74 and $\geqslant 13.5 ; S_{\mathrm{PO}_{2}} / F_{\mathrm{iO}_{2}}: 0.61$ and $\leqslant 185$; platelets: 0.59 and $\leqslant 196 \times 10^{9} \cdot \mathrm{L}^{-1}$; age: 0.64 and $\geqslant 37.5$ years; lactate: 0.63 and $\geqslant 2.45 \mathrm{mmol} \cdot \mathrm{L}^{-1}$; sodium: 0.60 and $\leqslant 130.5 \mathrm{mmol} \cdot \mathrm{L}^{-1}$. Hospital mortality in medical patients, based on the number of single risk factors with adverse levels based on these thresholds (OPALS: oxygen $\left(\mathrm{S}_{\mathrm{PO}_{2}} / F_{\mathrm{iO}_{2}}\right)$, platelets, age, lactate and sodium), is shown in figure 2. AUC for this OPALS score in medical patients was 0.78 with an optimal threshold $\geqslant 2.5$.

\section{Critical care interventions and mortality in the medical group}

Compared with overall survival to hospital discharge in the medical group, the requirement for ventilatory or cardiovascular support was associated with worse survival (table 3). One patient out of nine who was 


\section{TABLE 4 Medical patients: univariate predictors of hospital mortality}

\begin{tabular}{|c|c|c|c|}
\hline Variable & $\mathbf{N}$ & OR $(95 \% \mathrm{CI})$ & $\mathrm{p}$-value \\
\hline Age years & 167 & $1.02(1.01-1.04)$ & 0.005 \\
\hline Female (ref.) & 167 & $0.914(0.481-7.738)$ & 0.784 \\
\hline PH group & 167 & & 0.704 \\
\hline 1 Pulmonary arterial hypertension & & Ref. & \\
\hline $2 \mathrm{PH}$ due to left heart disease & & $1.568(0.303-8.120)$ & 0.592 \\
\hline $3 \mathrm{PH}$ due to lung disease/hypoxia & & $1.307(0.376-4.541)$ & 0.674 \\
\hline 4 Chronic thromboembolic $\mathrm{PH}$ & & $0.882(0.359-2.169)$ & 0.785 \\
\hline 5 Unclear/multifactorial & & $2.195(0.656-7.350)$ & 0.202 \\
\hline Admission reason ${ }^{\#}$ & 167 & & 0.03 \\
\hline Heart failure & & Ref. & Ref. \\
\hline Respiratory failure/chest sepsis & & $0.554(0.254-1.208)$ & 0.138 \\
\hline Sepsis (other) & & $0.356(0.099-1.277)$ & 0.113 \\
\hline Pulmonary embolism & & $0.800(0.150-4.274)$ & 0.794 \\
\hline Arrhythmia & & $0.133(0.028-0.646)$ & 0.012 \\
\hline Renal failure & & $0.267(0.066-1.079)$ & 0.064 \\
\hline Other & & $0.200(0.051-0.779)$ & 0.020 \\
\hline ISWD & 102 & $0.998(0.995-1.001)$ & 0.242 \\
\hline APACHE II & 137 & $1.180(1.095-1.270)$ & $<0.001$ \\
\hline Heart rate & 166 & $1.011(0.995-1.028)$ & 0.190 \\
\hline mSAP mmHg & 163 & $0.983(0.964-1.003)$ & 0.100 \\
\hline Respiratory rate & 167 & $1.046(1.005-1.087)$ & 0.026 \\
\hline $\mathrm{S}_{\mathrm{pO}_{2}} \%$ & 167 & $0.984(0.939-1.030)$ & 0.482 \\
\hline$F_{\mathrm{iO}_{2}}$ & 165 & $5.896(1.445-24.061)$ & 0.013 \\
\hline$P_{\mathrm{aO}_{2}} \mathrm{kPa}$ & 142 & $0.959(0.888-1.036)$ & 0.292 \\
\hline $\mathrm{S}_{\mathrm{pO}_{2}} / F_{\mathrm{iO}_{2}}$ ratio & 134 & $0.997(0.993-1.001)$ & 0.114 \\
\hline$P_{\mathrm{aO}_{2}} / F_{\mathrm{iO}_{2}}$ ratio & 134 & $0.983(0.959-1.008)$ & 0.187 \\
\hline Central venous pressure $\mathrm{mmHg}$ & 54 & $1.096(1.021-1.177)$ & 0.011 \\
\hline Glasgow Coma Scale & 138 & $0.727(0.539-0.980)$ & 0.036 \\
\hline $\mathrm{pH}$ & 148 & $0.007(0.000-0.393)$ & 0.016 \\
\hline Base excess $\mathrm{mEq} \cdot \mathrm{L}^{-1}$ & 147 & $0.933(0.886-0.984)$ & 0.01 \\
\hline Lactate $\mathrm{mmol} \cdot \mathrm{L}^{-1}$ & 141 & $1.425(1.117-1.817)$ & 0.004 \\
\hline Serum sodium mmol. $\mathrm{L}^{-1}$ & 157 & $0.937(0.884-0.993)$ & 0.027 \\
\hline Serum urea mmol. $\mathrm{L}^{-1}$ & 156 & $12.7(4.37-80.2)$ & 0.001 \\
\hline Serum creatinine $\mu \mathrm{mol} \cdot \mathrm{L}^{-1}$ & 155 & $1.005(1.001-1.009)$ & 0.013 \\
\hline Total bilirubin $\mu \mathrm{mol} \cdot \mathrm{L}^{-1}$ & 143 & $6.08(2.97-19.8)$ & 0.022 \\
\hline AST IU. $L^{-1}$ & 123 & $0.999(0.995-1.002)$ & 0.456 \\
\hline CRP $\mathrm{mg} \cdot \mathrm{L}^{-1}$ & 141 & $1.005(1.000-1.009)$ & 0.054 \\
\hline Haemoglobin $\mathrm{g} \cdot \mathrm{L}^{-1}$ & 157 & $0.990(0.978-1.003)$ & 0.123 \\
\hline Platelets $\times 10^{9} \cdot \mathrm{L}^{-1}$ & 156 & $0.997(0.994-1.000)$ & 0.033 \\
\hline Right atrial pressure $\mathrm{mmHg}$ & 109 & $1.044(0.990-1.101)$ & 0.108 \\
\hline Mean PAP mmHg & 114 & $1.005(0.973-1.037)$ & 0.775 \\
\hline PAWP $\mathrm{mmHg}$ & 102 & $1.000(0.919-1.088)$ & 1.000 \\
\hline Cardiac output L.min ${ }^{-1}$ & 110 & $0.909(0.743-1.112)$ & 0.352 \\
\hline Cardiac index L. $\min ^{-1} \cdot \mathrm{m}^{-2}$ & 104 & $0.838(0.584-1.201)$ & 0.335 \\
\hline PVR dyn $\cdot \mathrm{s}^{-1} \cdot \mathrm{cm}^{-5}$ & 99 & $1.000(0.999-1.001)$ & 0.482 \\
\hline $\mathrm{S}_{\mathrm{vO}_{2}} \%$ & 102 & 0.966 (0.929-1.005) & 0.087 \\
\hline
\end{tabular}

Variables either at most recent review prior to admission (Right heart catheter data, ISWD at 2 weeks to 18 months prior to admission) or at critical care admission (e.g. laboratory tests and physiological observations). Bold indicates statistical significance. PH: pulmonary hypertension; ISWD: incremental shuttle walking distance; APACHE II: Acute Physiology and Chronic Health Evaluation II; mSAP: mean systemic arterial pressure; $S_{\mathrm{pO}_{2}}$ : oxygen saturation measured by pulse oximetry; $F_{\mathrm{iO}_{2}}$ : inspiratory oxygen fraction; $P_{\mathrm{aO}_{2}}$ : arterial oxygen tension; AST: aspartate aminotransferase; CRP: C-reactive protein; PAP: pulmonary artery pressure; PAWP: pulmonary arterial wedge pressure; PVR: pulmonary vascular resistance; $\mathrm{S}_{\mathrm{vO}_{2}}$ : mixed venous oxygen saturation. "\# : main indication for critical care admission.

invasively ventilated survived to hospital discharge; however, this patient survived 17 months post-discharge and was in WHO FC II at initial follow-up. $50 \%$ of the 22 patients who required RRT survived to be discharged home. 


\begin{tabular}{|c|c|c|}
\hline Variable & OR $(95 \% \mathrm{CI})$ & p-value \\
\hline \multicolumn{3}{|l|}{ Model $1^{\#}$} \\
\hline APACHE II per point & $1.148(1.052-1.253)$ & 0.002 \\
\hline Sodium $\mathrm{mmol} \cdot \mathrm{L}^{-1}$ & $0.927(0.864-0.996)$ & 0.038 \\
\hline Platelets $\times 10^{9} \cdot \mathrm{L}^{-1}$ & $0.997(0.993-1.000)$ & 0.052 \\
\hline $\mathrm{S}_{\mathrm{pO}_{2}} / F_{\mathrm{iO}_{2}}$ ratio & $0.997(0.992-1.001)$ & 0.104 \\
\hline Bilirubin IU. $\mathrm{L}^{-1}$ & $0.997(0.970-1.024)$ & 0.806 \\
\hline Creatinine $\mu \mathrm{mol} \cdot \mathrm{L}^{-1}$ & $0.999(0.995-1.004)$ & 0.790 \\
\hline Lactate $\mathrm{mmol} \cdot \mathrm{L}^{-1}$ & $1.292(0.983-1.700)$ & 0.067 \\
\hline \multicolumn{3}{|l|}{ Model $2^{\pi}$} \\
\hline Age years & $1.045(1.018-1.073)$ & 0.001 \\
\hline Sodium $\mathrm{mmol} \cdot \mathrm{L}^{-1}$ & $0.901(0.836-0.971)$ & 0.007 \\
\hline Platelets $\times 10^{9} \cdot \mathrm{L}^{-1}$ & $0.996(0.993-1.000)$ & 0.038 \\
\hline $\mathrm{S}_{\mathrm{pO}_{2}} / F_{\mathrm{iO}_{2}}$ ratio & 0.995 (0.991-0.999) & 0.020 \\
\hline Total bilirubin $\mu \mathrm{mol} \cdot \mathrm{L}^{-1}$ & $0.993(0.966-1.021)$ & 0.620 \\
\hline Creatinine $\mu \mathrm{mol} \cdot \mathrm{L}^{-1}$ & $1.002(0.998-1.006)$ & 0.333 \\
\hline Lactate $\mathrm{mmol} \cdot \mathrm{L}^{-1}$ & $1.562(1.181-2.066)$ & 0.002 \\
\hline
\end{tabular}

APACHE II: Acute Physiology and Chronic Health Evaluation II; $\mathrm{S}_{\mathrm{pO}_{2}}$ : oxygen saturation measured by pulse oximetry; $F_{\mathrm{iO}_{2}}$ : inspiratory oxygen fraction. " : incorporating APACHE ${ }^{\prime \prime}$; ${ }^{\text {? }}$ : excluding APACHE II.
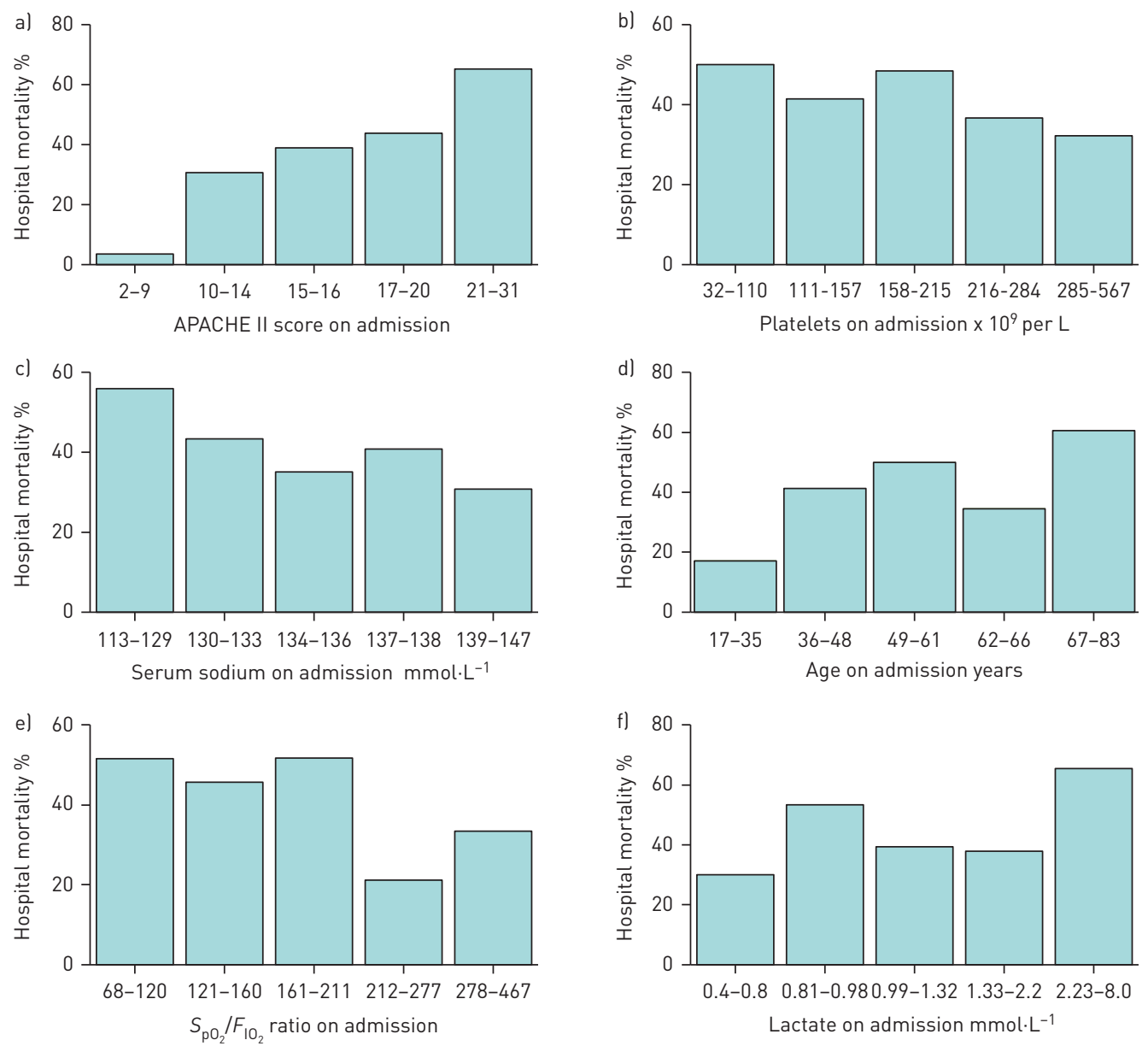

FIGURE 1 Histograms demonstrating in-hospital mortality in medical patients according to quintiles of a) APACHE II (Acute Physiology and Chronic Health Evaluation II) score, b) platelet count, c) serum sodium level, d) age, e) oxygen saturation measure by pulse oximetry $\left(\mathrm{S}_{\mathrm{PO}_{2}}\right) /$ inspiratory oxygen fraction $\left(F_{\mathrm{iO}_{2}}\right)$ ratio and f) lactate. Each bar represents $20 \%$ of the population for that parameter. 
FIGURE 2 OPALS score. Risk of hospital mortality in medical patients based on number of adverse single risk factors. OPALS: oxygen loxygen saturation measured by pulse oximetry/inspiratory oxygen fraction ratiol $\leqslant 185$; platelets $\leqslant 196 \times 10^{9} \cdot \mathrm{L}^{-1} ;$ age $\geqslant 37.5$ years; lactate $\geqslant 2.45 \mathrm{mmol} \cdot \mathrm{L}^{-1} ;$ sodium $\leqslant 130.5 \mathrm{mmol} \cdot \mathrm{L}^{-1}$ ).

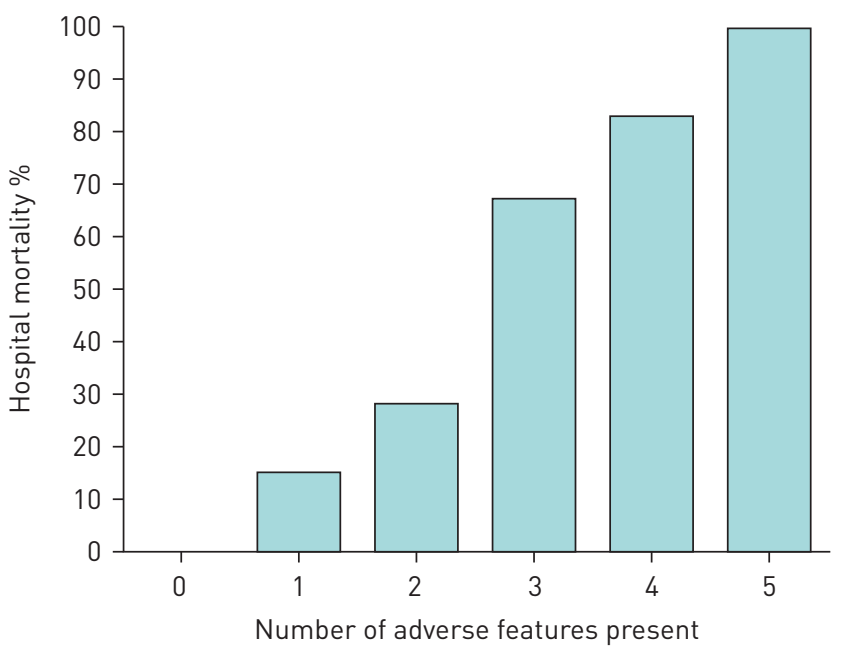

$\begin{array}{ccccccc}\text { Adverse features } & 0 & 1 & 2 & 3 & 4 & 5 \\ \text { Patients } & 6 & 20 & 53 & 43 & 12 & 2\end{array}$

\section{Surgical and obstetric patients}

Three $(6 \%)$ out of 50 patients admitted following surgery died before hospital discharge. Two of the patients had undergone expedited complex abdominal cancer surgery and one patient presented for an emergency laparotomy. All three patients had severe cardio-respiratory comorbidities, two patients had established multiple organ failure prior to surgery and one patient suffered an intra-operative complication. Two out of 25 patients admitted for obstetric reasons died before hospital discharge; both patients had severe $\mathrm{PAH}$, one of whom had suboptimal compliance with $\mathrm{PAH}$ medications during pregnancy while the other patient presented immediately following delivery with previously undiagnosed $\mathrm{PH}$. Both patients deteriorated despite aggressive management including intravenous prostanoid therapy.

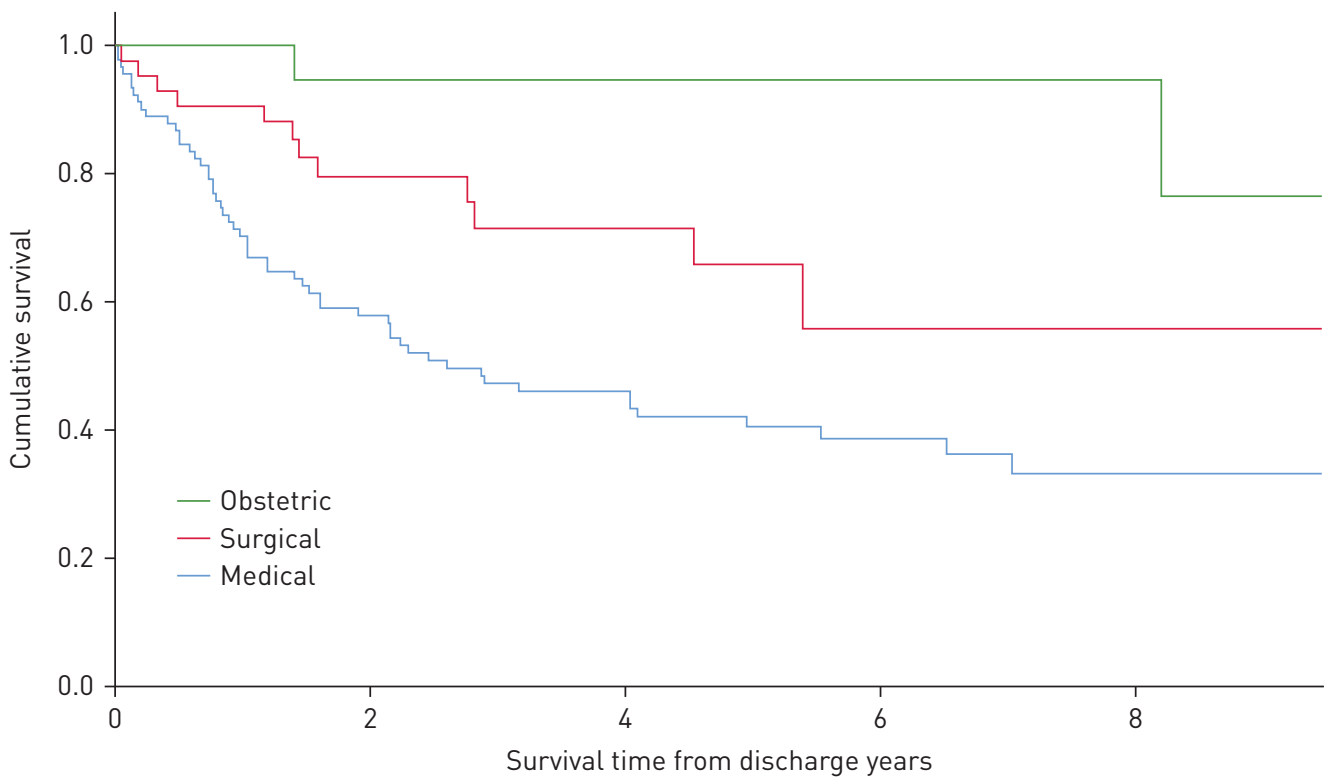

Patients at risk

$\begin{array}{rrrrrr}\text { Obstetric } & 18 & 15 & 10 & 6 & 5 \\ \text { Surgical } & 40 & 22 & 13 & 5 & 3 \\ \text { Medical } & 86 & 46 & 31 & 16 & 7\end{array}$

FIGURE 3 Long-term survival of patients discharged from hospital following their first presentation to critical care. $p=0.008$ medical versus surgical, $p=0.001$ medical versus obstetric, $p=0.073$ surgical versus obstetric. 


\section{Long-term survival}

The overall observed survival rates after critical care admission were $65 \%$ at 90 days, $55 \%$ at 1 year and $31 \%$ at 5 years. For the medical cohort, these survival rates were $55 \%, 41 \%$ and $23 \%$, respectively (table 3 ). The median (95\% CI) survival time from first critical care admission was 17.9 months (546 (range 255-838) days) overall and 7.9 months (241 (54-428) days) for medical patients. For those patients surviving to hospital discharge after first critical care admission, Kaplan-Meier analysis demonstrated superior 1- and 5-year survival in the surgical and obstetric groups (90\% and 100\% at 1 year, $63 \%$ and $94 \%$ at 5 years) when compared with the medical group ( $69 \%$ and $37 \%$, respectively; $\mathrm{p}=0.012$ medical versus surgical, $\mathrm{p}=0.004$ medical versus obstetric, $\mathrm{p}=0.07$ surgical versus obstetric) (figure 3 ).

\section{Functional outcomes in critical care survivors}

Comparison of the mean (95\% CI) ISWD prior to admission versus nearest 6- and 12-months' follow-up in hospital survivors for the medical group showed no statistical difference on paired samples t-testing (baseline $233 \mathrm{~m}$ (95\% CI 186-281) versus 6-months follow-up $206 \mathrm{~m}$ (95\% CI 164-248), $\mathrm{p}=0.063$ or versus 12-months follow-up $227 \mathrm{~m}$ (95\% CI 181-273), $\mathrm{p}=0.941)$. This lack of change was also observed across the surgical group, while there were insufficient data available for the obstetric group. Similarly, there was no significant difference in the WHO FC before and after critical care admission in the medical group using Wilcoxon signed-rank test (median (range) WHO FC 3 (2-4) at both time-points, $\mathrm{p}=0.197$ ). This lack of change was also observed across the obstetric and surgical populations.

\section{Discussion}

To our knowledge this is the largest study, to date, of patients with well-characterised $\mathrm{PH}$ who have been treated in a critical care setting. We have demonstrated that medical patients have more severely abnormal physiological and laboratory markers than surgical/obstetric patients and have poorer short- and longer term prognosis. We have also identified several important markers of in-hospital mortality in patients admitted due to medical decompensation. Although only a very small proportion of patients receiving invasive ventilation were discharged from hospital, $50 \%$ of patients who required RRT left hospital alive. Finally, we were unable to find a significant difference between pre-admission and post-discharge exercise capacity and WHO FC, suggesting that patients who survive to hospital discharge may regain a similar functional state following a critical care admission.

\section{Survival}

SzTRYMF et al. [13] previously studied $46 \mathrm{PAH}$ patients with right heart failure and observed $41 \%$ ICU mortality. Subsequently, HuYnh et al. [11] reported 30\% ICU and 40\% 6-month mortality in $99 \mathrm{PH}$ patients while KURZYNA et al. [18] and CAMPO et al. [19] observed 32\% and 48\% in-hospital mortalities in their studies of 37 and 29 critically ill PH patients, respectively. SAYDAIN et al. [12] studied 53 patients (largely composed of PH secondary to lung or left heart disease) and observed hospital mortality of $26 \%$ to $36 \%$, while TSAPENKo et al. [14] reported hospital mortality of $48 \%$ in a $\mathrm{PH}$ patient cohort largely secondary to lung disease. Critical care $(28 \%)$ and in-hospital (41\%) mortality of our patients admitted to critical care for medical reasons are consistent with these previous reports and highlight the significant mortality associated with decompensation in patients with pre-existing PH. Of note, all admissions to critical care were deemed appropriate following a multidisciplinary discussion between $\mathrm{PH}$ and ICU physicians. Survival figures therefore reflect a carefully selected patient cohort, rather than an undifferentiated $\mathrm{PH}$ population. Although only $38 \%$ of medical patients were admitted due to isolated right heart failure (i.e. progressive right heart failure with no obvious precipitant), the majority of other medical patients had either a precipitant of right heart failure (e.g. sepsis, respiratory failure or arrhythmia) or a complication of right heart failure (e.g. renal failure).

Hospital mortality for surgical and obstetric patients was significantly lower (6\% and $8 \%$ ) than in medical patients and is in keeping with previous reports [8, 9, 20]. PRICE et al. [8] studied 28 patients with $\mathrm{PH}$ who underwent non-cardiac and non-obstetric surgery and observed a peri-operative death rate of $7 \%$. MEYER et al. [9] subsequently studied 114 patients with $\mathrm{PAH}$ who underwent non-cardiac and non-obstetric surgery and reported an overall mortality of $3.5 \%$, with emergency surgery being associated with a significantly higher death rate than planned surgery (15\% versus $2 \%$ ). Despite outcomes in pregnancy having improved over recent years, the peripartum period is recognised as posing a significant risk and two patients with severe PAH (one of whom presented following delivery) died post-partum despite aggressive management $[10,20]$.

\section{Prognostic markers}

RV failure in decompensated PH leads to low cardiac output (forward failure) and impaired venous return (backward failure) $[21,22]$. We identified a number of significant prognostic markers at univariate analysis 
which reflect these two processes: low cardiac output: acid-base disturbance (base excess, lactate); impaired venous return: central venous pressure and hepatic congestion (bilirubin); and a combination of the two processes: renal impairment (urea, creatinine). We also identified several independent additional prognostic markers: lower $S_{\mathrm{pO}_{2}} / F_{\mathrm{IO}_{2}}$ ratio, serum sodium, and platelet count and higher age, lactate and APACHE II score.

Hypoxia in $\mathrm{PH}$ results in worsened pulmonary vasoconstriction with subsequent detrimental effects on RV function [21, 23]. Hypoxaemia at rest or during exercise has been shown to be predictive of lower survival duration in stable PAH patients [24]. Our data suggests that this observation can be extended to the critical care population, where lower $S_{\mathrm{pO}_{2}} / F_{\mathrm{iO}_{2}}$ ratios at critical care admission indicated higher hospital mortality. Our observation that lower serum sodium predicted acute mortality is consistent with reports of reduced longer term survival in stable $\mathrm{PH}$ patients [25] and increased short-term mortality in hospitalised $\mathrm{PAH}$ patients with right ventricular failure $[13,19]$. Hyponatraemia is a hallmark of cardiorenal syndrome and may result from both low cardiac output and congestive RV failure [26]. The present study is the first to report prognostic utility of the admission platelet count in ICU patients. Previous reports in stable patients have described an association with right atrial pressure and mixed venous oxygen saturations [27] and demonstrated that the degree of thrombocytopenia may be an independent predictor of 1-year survival in patients with severe $\mathrm{PH}$ [28]. Thrombocytopenia may occur due to reduced thrombopoietin production, bone marrow suppression, increased sequestration and platelet activation, aggregation from vascular stasis in an altered circulatory environment and the effects of both targeted therapies and associated underlying disease processes $[29,30]$. We were unable to distinguish between patients who had received intravenous prostanoid therapy on the ward prior to critical care admission from those who had commenced it after admission to critical care and so could not draw any conclusions regarding its possible contribution to the observed thrombocytopenia.

Previous studies have demonstrated the prognostic utility of acute physiological scores in $\mathrm{PH}$ patients on admission, including APACHE II [11], SAPS II [13] or SOFA scores [12]. APACHE II is comprised of age plus 12 physiological and laboratory measurements: arterial oxygen tension; temperature; mean arterial pressure; $\mathrm{pH}$; heart rate; respiratory rate; sodium, potassium and creatinine levels; haematocrit; white blood cell count; and Glasgow Coma Scale. Many of these parameters were predictors of outcome at univariate analysis. When APACHE II was excluded from the multivariate model, age, serum sodium, platelet count, $S_{\mathrm{pO}_{2}} / F_{\mathrm{iO}_{2}}$ ratio and lactate were independent predictors, suggesting that these components of APACHE II are of particular importance. A simple exploratory scoring system which can be quickly calculated at the bedside in medical patients using the five independent single risk factors, OPALS (oxygen $\left(S_{\mathrm{pO}_{2}} / F_{\mathrm{iO}_{2}}\right) \leqslant 185$, platelets $\leqslant 196 \times 10^{9} \cdot \mathrm{L}^{-1}$, age $\geqslant 37.5$ years, lactate $\geqslant 2.45 \mathrm{mmol} \cdot \mathrm{L}^{-1}$, sodium $\left.\leqslant 130.5 \mathrm{mmol} \cdot \mathrm{L}^{-1}\right)$, identified patients at increasing risk of mortality. The numbers of patients with 0 or 5 adverse factors was, however, very low and validation and further comparison with APACHE II in other large cohorts is required.

It was interesting to note that although markers of acute physiological deterioration predicted critical care outcomes, pulmonary haemodynamics did not. This may reflect the fact that right heart catheterisation is not repeated at routine intervals and so the most recent pulmonary haemodynamics may have been obtained some time before the critical care admission.

\section{Interventions}

The need for advanced organ support in the medical cohort was associated with poorer survival, consistent with more severely unwell patients requiring these treatments. Of note, out of nine medical patients who received invasive ventilation, only three (33\%) survived to critical care discharge and a single patient $(11 \%)$ survived to hospital discharge, suggesting that invasive ventilation is likely appropriate only in selected cases. It is interesting to observe that $50 \%$ of patients who required RRT survived to hospital discharge. HADDAD et al. [31] previously identified acute kidney injury in PAH patients hospitalised with right heart failure as an important predictor of poor outcome. Acute kidney injury in patients with $\mathrm{PH}$ is associated with renal venous congestion [32]; RRT could potentially offer significant beneficial effects on management of volume status and right ventricular function in $\mathrm{PH}$ patients [33, 34]. There are very limited data describing the use of acute RRT in PH patients [31]. Our data would suggest that RRT should be considered in patients with acute decompensation.

\section{Longer term survival and functional outcome}

This is the first study to report longer term outcomes in $\mathrm{PH}$ patients admitted to a critical care unit. 1 -year survival of $40.7 \%$ in medical patients suggests that critical care intervention may enable additional quantity of life in a proportion of patients. Functional follow-up data (WHO FC and ISWD) of survivors suggests that many survivors to discharge regain similar levels of functioning as compared with their pre-admission state. 


\section{Limitations}

Due to the long timescale of the study there were missing data in variables relating to pre-admission investigations, making firm conclusions regarding their prognostic importance, or lack of, difficult. The single-centre and retrospective nature of the study design may impede generalisability of the findings. Nevertheless, these data represent long-term experience from a large PH referral centre and our findings are generally consistent with previous smaller studies $[11,13,19]$.

\section{Conclusion}

Medical patients admitted to a critical care unit because of acute decompensation have more severe physiological and laboratory perturbations and poorer survival than patients admitted following surgical or obstetric interventions. Higher APACHE II score, age and lactate, and lower $S_{\mathrm{pO}_{2}} / F_{\mathrm{iO}_{2}}$, platelet count and sodium level are important predictors of hospital discharge. Invasive ventilation in medically decompensated patients is associated with a low chance of hospital survival whereas $50 \%$ of patients receiving RRT leave hospital alive. In patients who survive to discharge, pre-admission levels of functional status may be achieved. These data have clinical utility in guiding decisions regarding critical care admission and intensity of interventions in patients with known $\mathrm{PH}$.

Conflict of interest: K. Bauchmuller has nothing to disclose. R. Condliffe reports honoraria for lecturing and advisory boards from Actelion and MSD outside the submitted work. J. Southern has nothing to disclose. C. Billings has nothing to disclose. A. Charalampopoulos has nothing to disclose. C.A. Elliot received lecture and consultancy fees from Actelion, Bayer and GSK pharmaceuticals. A. Hameed has nothing to disclose. D.G. Kiely reports grants, personal fees and other support from Actelion, Bayer and GSK, and personal fees and other support from MSD, outside the submitted work. I. Sabroe has nothing to disclose. A.A.R. Thompson reports an Intermediate Clinical Fellowship (FS/ 18/13/3328) from the British Heart Foundation during the conduct of the study and support for travel to attend educational meetings from Actelion Pharmaceuticals Ltd outside the submitted work. A. Raithatha has nothing to disclose. G.H. Mills has nothing to disclose.

Support statement: This study was supported by British Heart Foundation grant FS/18/13/3328. Funding information for this article has been deposited with the Crossref Funder Registry.

\section{References}

1 Galie N, Humbert M, Vachiery JL, et al. 2015 ESC/ERS Guidelines for the diagnosis and treatment of pulmonary hypertension: The Joint Task Force for the Diagnosis and Treatment of Pulmonary Hypertension of the European Society of Cardiology (ESC) and the European Respiratory Society (ERS): Endorsed by: Association for European Paediatric and Congenital Cardiology (AEPC), International Society for Heart and Lung Transplantation (ISHLT). Eur Heart J 2016; 37: 67-119.

2 Kiely DG, Elliot CA, Sabroe I, et al. Pulmonary hypertension: diagnosis and management. BMJ 2013; 346: f2028.

3 Condliffe R, Kiely DG. Critical care management of pulmonary hypertension. BJA Education 2017; 17: 228-234.

4 Condliffe R, Kiely DG, Gibbs JS, et al. Improved outcomes in medically and surgically treated chronic thromboembolic pulmonary hypertension. Am J Respir Crit Care Med 2008; 177: 1122-1127.

5 Humbert M, Sitbon O, Chaouat A, et al. Survival in patients with idiopathic, familial, and anorexigen-associated pulmonary arterial hypertension in the modern management era. Circulation 2010; 122: 156-163.

6 Hoeper MM, Benza RL, Corris P, et al. Intensive care, right ventricular support and lung transplantation in patients with pulmonary hypertension. Eur Respir J 2019; 53: 1801906.

7 Price LC, Dimopoulos K, Marino P, et al. The CRASH report: emergency management dilemmas facing acute physicians in patients with pulmonary arterial hypertension. Thorax 2017; 72: 1035-1045.

8 Price LC, Montani D, Jais X, et al. Noncardiothoracic nonobstetric surgery in mild-to-moderate pulmonary hypertension. Eur Respir J 2010; 35: 1294-1302.

9 Meyer S, McLaughlin VV, Seyfarth HJ, et al. Outcomes of noncardiac, nonobstetric surgery in patients with PAH: an international prospective survey. Eur Respir J 2013; 41: 1302-1307.

10 Bedard E, Dimopoulos K, Gatzoulis MA. Has there been any progress made on pregnancy outcomes among women with pulmonary arterial hypertension? Eur Heart J 2009; 30: 256-265.

11 Huynh TN, Weigt SS, Sugar CA, et al. Prognostic factors and outcomes of patients with pulmonary hypertension admitted to the intensive care unit. J Crit Care 2012; 27: 739.e7-13.

12 Saydain G, Awan A, Manickam P, et al. Pulmonary hypertension an independent risk factor for death in intensive care unit: correlation of hemodynamic factors with mortality. Clin Med Insights Circ Respir Pulm Med 2015; 9: $27-33$.

13 Sztrymf B, Souza R, Bertoletti L, et al. Prognostic factors of acute heart failure in patients with pulmonary arterial hypertension. Eur Respir J 2010; 35: 1286-1293.

14 Tsapenko MV, Herasevich V, Mour GK, et al. Severe sepsis and septic shock in patients with pre-existing non-cardiac pulmonary hypertension: contemporary management and outcomes. Crit Care Resusc 2013; 15: 103-109.

15 Rush B, Biagioni BJ, Berger L, et al. Mechanical ventilation outcomes in patients with pulmonary hypertension in the United States: a national retrospective cohort analysis. J Intensive Care Med. 2017; 32: 588-592.

16 Hurdman J, Condliffe R, Elliot CA, et al. ASPIRE registry: Assessing the Spectrum of Pulmonary hypertension Identified at a REferral centre. Eur Respir J 2012; 39: 945-955.

17 Simonneau G, Gatzoulis MA, Adatia I, et al. Updated clinical classification of pulmonary hypertension. J Am Coll Cardiol 2013; 62: D34-D41.

18 Kurzyna M, Zylkowska J, Fijalkowska A, et al. Characteristics and prognosis of patients with decompensated right ventricular failure during the course of pulmonary hypertension. Kardiol Pol 2008; 66: 1033-1039. 
Campo A, Mathai SC, Le Pavec J, et al. Outcomes of hospitalisation for right heart failure in pulmonary arterial hypertension. Eur Respir J 2011; 38: 359-367.

20 Kiely DG, Condliffe R, Webster V, et al. Improved survival in pregnancy and pulmonary hypertension using a multiprofessional approach. BJOG 2010; 117: 565-574.

21 Hoeper MM, Granton J. Intensive care unit management of patients with severe pulmonary hypertension and right heart failure. Am J Respir Crit Care Med 2011; 184: 1114-1124.

22 Wilcox SR, Kabrhel C, Channick RN. Pulmonary hypertension and right ventricular failure in emergency medicine. Ann Emerg Med 2015; 66: 619-628.

23 Greyson CR. The right ventricle and pulmonary circulation: basic concepts. Rev Esp Cardiol 2010; 63: 81-95.

24 Khirfan G, Naal T, Abuhalimeh B, et al. Hypoxemia in patients with idiopathic or heritable pulmonary arteria hypertension. PLoS One 2018; 13: e0191869.

25 Forfia PR, Mathai SC, Fisher MR, et al. Hyponatremia predicts right heart failure and poor survival in pulmonary arterial hypertension. Am I Respir Crit Care Med 2008; 177: 1364-1369.

26 Jentzer JC, Mathier MA. Pulmonary hypertension in the intensive care unit. J Intensive Care Med 2016; 31: 369-385.

27 Chin KM, Channick RN, de Lemos JA, et al. Hemodynamics and epoprostenol use are associated with thrombocytopenia in pulmonary arterial hypertension. Chest 2009; 135: 130-136.

28 Mojadidi MK, Goodman-Meza D, Eshtehardi P, et al. Thrombocytopenia is an independent predictor of mortality in pulmonary hypertension. Heart Lung 2014; 43: 569-573.

29 Remkova A, Simkova I, Valkovicova T, et al. Platelet abnormalities in adults with severe pulmonary arterial hypertension related to congenital heart defects (Eisenmenger syndrome). Blood Coagul Fibrinolysis 2016; 27: 925-929.

30 Vrigkou E, Tsangaris I, Bonovas S, et al. Platelet and coagulation disorders in newly diagnosed patients with pulmonary arterial hypertension. Platelets 2019; 30: 646-651.

31 Haddad F, Fuh E, Peterson T, et al. Incidence, correlates, and consequences of acute kidney injury in patients with pulmonary arterial hypertension hospitalized with acute right-side heart failure. J Card Fail 2011; 17: 533-539.

32 Damman K, Navis G, Smilde TD, et al. Decreased cardiac output, venous congestion and the association with renal impairment in patients with cardiac dysfunction. Eur J Heart Fail 2007; 9: 872-878.

33 Ventetuolo CE, Klinger JR. Management of acute right ventricular failure in the intensive care unit. Ann Am Thorac Soc 2014; 11: 811-822.

34 Olsson KM, Halank M, Egenlauf B, et al. Decompensated right heart failure, intensive care and perioperative management in patients with pulmonary hypertension: Updated recommendations from the Cologne Consensus Conference 2018. Int J Cardiol 2018; 272S: 46-52. 